\title{
Conférences Bernard Nominé
}

\section{Résumé}

Ce texte est le résultat du travail de deux conférences dictées au Forum du Champ lacanien de Fortaleza, en février 2019, lors de l'ouverture des séminaires sur la topologie. Au départ, Bernard Nominé nous parle de la rencontre de Lacan avec le nœud borroméen et des répercussions qui en découlent par rapport à la psychanalyse, mettant l'accent sur des questions théoriques jusque-là mal comprises et qui pourraient être ré-entrelacés tout au long de sa pratique. Ensuite, l'auteur examine le lien entre le nœud borroméen et l'écriture de la clinique, nous rappelant que souvent nos catégories de névrose, psychose et perversion ne suffisent pas à extraire la logique de certains cas. Par conséquent, on doit savoir manier la chaîne borroméenne, ses principales failles, ses suites et ses éventuelles réparations. Pour ce faire, il faut aller plus loin dans ce que l'auteur appelle « le solfège des noeuds ».

\section{Mots-clés :}

Jacques Lacan ; Nœud borroméen ; Inconscient.

\section{Bernard Nominé's Conferences}

\begin{abstract}
This text is the result of the work of two conferences given at the Fortaleza Forum of the Lacanian Field in February 2019, during the opening of the seminars about topology. At first, Bernard Nominé spoke about Lacan's encounter with the Borromean knot and its repercussions on psychoanalysis, pointing out previously misunderstood theoretical issues that could be re-enlaced throughout his practice. Then the author focused on the relation between the Borromean knot and the writing of the clinic, reminding us that our categories of neurosis, psychosis and perversion are often not sufficient to extract the logic of certain cases. Thus, it is necessary to know the handling of the Borromean chain, its main failures, consequences and possible repairs. To do so, it is necessary to go further in what the author calls "Borromean knot solfeggio".
\end{abstract}

\section{Keywords:}

Jacques Lacan; Borromean knot; Unconscious. 


\title{
Conferências de Bernard Nominé
}

\section{Resumo}

Este texto é resultado do trabalho de duas conferências proferidas no Fórum do Campo Lacaniano de Fortaleza, em fevereiro de 2019, na ocasião de abertura dos seminários sobre topologia. No primeiro momento, Bernard Nominé falou sobre o encontro de Lacan com o nó borromeano e suas repercussões na psicanálise, apontando questões teóricas antes mal-entendidas que puderam ser reenlaçadas ao longo de sua prática. Depois, o autor se deteve na relação do nó borromeano com a escrita da clínica, lembrando-nos de que nossas categorias de neurose, psicose e perversão muitas vezes não são suficientes para extrair a lógica de determinados casos. Assim, apresenta-se a necessidade de conhecer o manejo da cadeia borromeana, suas principais falhas, consequências e reparações possíveis. Para tanto, é preciso ir adiante naquilo que o autor denomina "solfejo do nó".

\author{
Palavras-chave: \\ Jacques Lacan; Nó borromeano; Inconsciente.
}

\section{Conferencias de Bernard Nominé}

Resumen

Este texto es el resultado del trabajo de dos conferencias impartidas en el Foro de Campo Lacaniano de Fortaleza, en febrero de 2019, en la ocasión de la apertura de los seminarios de topología. En el primer momento, Bernard Nominé habló sobre el encuentro de Lacan con el nudo borromeo y sus repercusiones en el psicoanálisis, señalando problemas teóricos previamente incomprendidos que pudieran entrelazarse a lo largo de su práctica. Luego, el autor pasó a relacionar el nudo borromeo con la escritura clínica, recordándonos que nuestras categorías de neurosis, psicosis y perversión a menudo no son suficientes para extraer la lógica de algunos casos. Así, se presenta la necesidad de conocer el manejo de la cadena borromea, sus principales problemas, consecuencias y posibles reparaciones. Para hacerlo, es necesario ir más allá en lo que el autor llama "solfeo del nudo borromeo".

\section{Palabras clave:}

Jacques Lacan; Nudo borromeo; Inconsciente. 


\section{La rencontre de Lacan avec le nœud borroméen ${ }^{1}$}

$(16 / 02 / 2019)$

C’est assez frappant de constater que très tôt dans son enseignement, dès 1953, Lacan a magistralement distingué trois dimensions avec lesquelles l'être parlant se repère : le Symbolique, l'Imaginaire et le Réel. Quand on y réfléchit bien et quand on relit ses séminaires, après-coup, on s'aperçoit qu'il a essayé d'articuler ces trois registres de différentes façons. La première c'est pour moi le schéma optique où il nous montre que ce qui du réel est invisible est remplacé par une image réelle dans le miroir sphérique et qu'ensuite cette image est traduite par le miroir plan qui figure l'Autre, donc le symbolique.

Une autre articulation, c'est le schéma $\mathrm{R}$ qui figure l'articulation du ternaire imaginaire et du ternaire symbolique au moyen d'un espace central qui figure le réel. Une troisième articulation c'est le graphe, ce parcours compliqué où il essaye de figurer comment le sujet se débrouille du réel avec l'outil de la parole qui s'articule en demande, puis au-delà de la demande l'espace du désir et tout ce qui s'échappe come réel, c'est-à-dire le reste de voix, le reste de regard... etc.

Mais il faut attendre 1972 pour que Lacan (1971-1972) trouve une façon de nouer le plus simplement du monde ces trois registres. C'est à l'époque du séminaire $\mathrm{Ou}$ pire... que Lacan nous livre une formule de son cru qu'il tient pour être la formule de la lettre d'amour, plus exactement la lettre d'(a) mur. Une lettre qui souligne qu'entre l'homme et la femme il y a le mur de l'amour. La formule est la suivante : "Je te demande de me refuser ce que je t'offre parce que c'est pas ça. » Il est très occupé à essayer d'écrire dans un graphe complexe la conjonction improbable de ces trois verbes quand on lui apprend qu'un certain Guilbaud, mathématicien de référence pour Lacan, vient de donner un cours sur la chaîne qui fait l'emblème des armoiries de la famille Borromée.

Aussitôt Lacan s'empare de ce qui lui semble lui aller comme une bague au doigt et le nœud borroméen ne le lâchera plus. Alors qu'a-t-il entrevu ? Il a entrevu que les trois verbes qui ne s'articulent pas naturellement dans sa formule un peu spéciale mais qui décrit bien la clinique de la vie amoureuse, ces trois verbes ne peuvent s'articuler qu'à la condition de poser que ce que je te demande, ce n'est pas ça que je veux, ce que je t'offre, ce n'est pas ça non plus que tu veux, et c'est donc au titre de ce que ce n'est pas ça, que tu as toutes les raisons de me le refuser. Si nous faisons figurer

\footnotetext{
1 Ce texte est le résultat de la combinaison de deux conférences de Bernard Nominé à Fortaleza, les 16 et 17 février 2019, à l'occasion de l'ouverture des Séminaires 2019 du Forum de Champ lacanien de Fortaleza. Une partie de la conférence a été supprimée, sous demande de l'auteur — celle qui évoquait des vignettes cliniques. (N.R.)
} 
chacun des trois verbes par un rond de ficelle, nous voyons que la phrase ne fait sens qu'à articuler les trois verbes autour d'un ce n'est pas ça central qui les fait tenir. Le fameux ce n'est pas ça figure la place de l'objet $a$ au centre du nœud. "C'est d'un nœud de sens que surgit l'objet a » (Lacan, 1971-1972, p. 64).

Figure 1

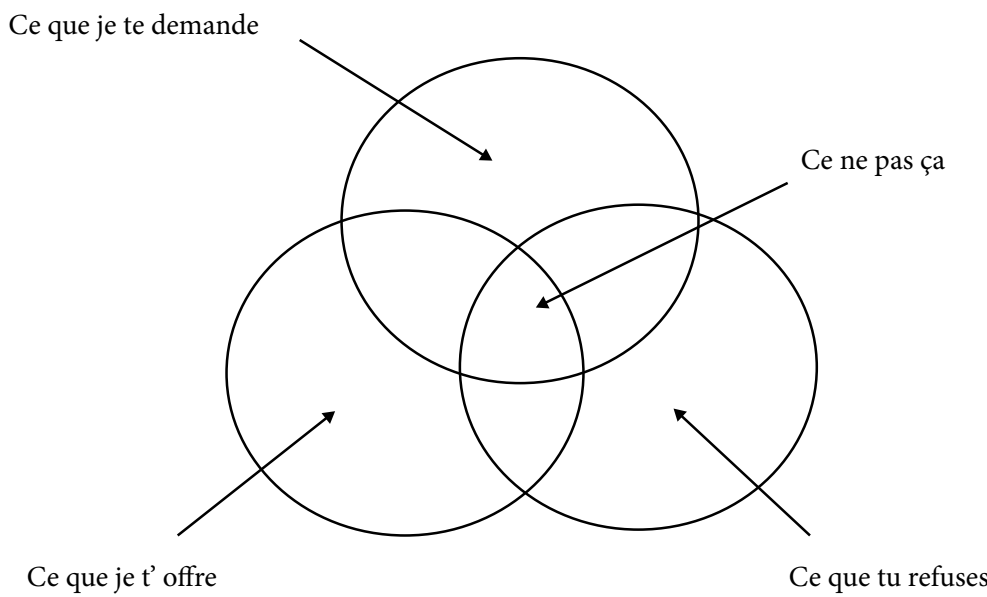

Je retiens donc de cet historique de l'invention de la théorie borroméenne de Lacan, trois choses essentielles :

1) Lacan a choisi la chaine borroméenne pour faire figurer un dire, pas n'importe lequel, un dire qui fait évènement, le dire de l'amour. Le nœud figure ce dire.

2) Dans ce dire qui a la structure de la chaine borroméenne trois ronds s'articulent de telle sorte que si l'on en ôte un, les deux autres se désolidarisent.

3) Mais n'oublions pas que les trois ronds s'articulent autour d'un objet qui n'est pas ça mais qui les concernent tous les trois.

A présent je vais vous faire part de ce que j’ai rencontré en travaillant cette question de la théorie borroméenne chez Lacan. C'est une série de surprises, de méprises entraperçues, bref des choses que je croyais assurées se défaisaient pour se renouer autrement. Je crois que cela tient à la difficulté d'élaboration d'une logique ternaire. Cela n'enlève rien à l'intérêt de ce moment passionnant de l'enseignement de Lacan. Il nous a légué un outil pour appuyer notre pensée sur la psychanalyse. C'est un outil génial mais ce n'est pas le chausse-pied qui permettrait de faire rentrer toutes les chevilles dans les petits trous. Restera toujours un quelque chose qui « n'est pas ça » qui nous pousse plus loin. C’est en cela qu'il nous est indispensable pour penser la psychanalyse. 
Premier point : Ce que j'ai présenté à Medellin, le nœud olympique qui est le contrepoint du nœud borroméen et dont Lacan nous dit dans Les non-dupes... que c'est le nœud de la névrose. Un des ronds vous manque et vous vous en sortez parce que les deux autres sont noués. En conséquence, les névrosés sont « increvables ». Lacan énonce ça très fortement mais il ne reviendra pas dessus ni pour l'infirmer ni pour le confirmer. Ça a pourtant des conséquences fondamentales à mon sens pour envisager la dynamique du transfert et pour l'acte analytique.

C’est sur la base de ce nœud olympique que Lacan envisage la phobie du petit Hans. Et si vous avez eu l'occasion d'étudier l'erreur du nœud en quoi consiste la réalité psychique de Joyce, vous verrez que Réel et Symbolique y sont noués entre eux, laissant l'imaginaire à la dérive. Ceci contredit la thèse selon laquelle dans la psychose les trois ronds sont libres entre eux, à moins qu'on considère qu'il n'est pas certain que Joyce soit fou.

Deuxième point : Quand on évoque la chaîne borroméenne, on situe le Réel comme l'un de ses éléments à côté du Symbolique et de l'Imaginaire. Mais ce que Lacan précise au fur et à mesure de son élaboration, c'est que le Réel se repère à la nécessité du trois. Autrement dit, le Réel, ce n'est pas seulement le rond du Réel, le Réel c'est le nœud et précisément le nœud à trois. C'est quelque chose que Lacan avance dans Les non-dupes et c'est quelque chose qui ne bougera pas. Il dira, par exemple dans le séminaire Le Sinthome: "A l'Imaginaire et au Symbolique, c'est-à-dire à des choses qui sont très étrangères, le Réel apporte l'élément qui peut les faire tenir ensemble. C'est quelque chose que je considère comme n'étant rien de plus que mon symptôme » (Lacan, 1975-1976, p. 81). Et il précise que ce symptôme est une réponse à l'invention de l'inconscient freudien. On touche là un point essentiel de l'invention lacanienne. En réponse à l'invention freudienne Lacan isole la catégorie du Réel qui se définit de rester en dehors de l'Imaginaire et en dehors du Symbolique mais d'être aussi ce qui les unit et qui donc, les fait participer à son caractère de réel puisqu'ils font partie de la chaîne.

\section{Le nœud à quatre}

Quand Lacan nous conduit à distinguer le rond du Réel et le Réel du nœud, il introduit une sorte de quatrième implicite. Car il nous est plus commode d'imaginer que le rond du Réel et le Réel du nœud ce sont deux entités différentes. Là, il nous faudrait revenir à la logique de Richard de Saint Victor dans son De Trinitate pour voir comment les théologiens se sont sortis de cette aporie, mais je crois bien me souvenir que Saint Victor récuse le quatre et s'en tient fermement au trois.

Ce n'est pas le fruit du hasard ni du fil de mes associations libres si en passant du trois au quatre j'en viens à faire référence à la fonction de Dieu-le-père.

Lacan nous y conduit forcément quand il remarque dans son séminaire RSI que chez Freud les trois consistances ne sont pas nouées, il lui faut inventer un 
quatrième terme : la réalité psychique qui n'est pas autre chose que le complexe d'Cidipe. Et pour Lacan (1974-1975, p. 78) cet CEdipe n'est pas si complexe que ça, c'est ce qu'il préfère appeler le Nom-du-Père, « ce qui ne veut rien dire que le Père comme Nom, le Père comme nommant. » Et Lacan (1974-1975, p. 79) précise : «Quand je dis le Nom-du-Père, ça veut dire qu'il peut y en avoir, comme dans le nœud borroméen, un nombre indéfini ».

En effet supposons trois ronds rouge jaune et vert non noués, on peut en faire une chaîne à condition d'utiliser un quatrième rond qui n'aura rien de particulier si ce n'est cette fonction de les réunir en chaîne. «Tout repose sur un qui, en tant que trou, communique sa consistance à tous les autres » (1974-1975, p. 79). A la fin de RSI Lacan parle du quatrième en tant que nomination.

Figure 2

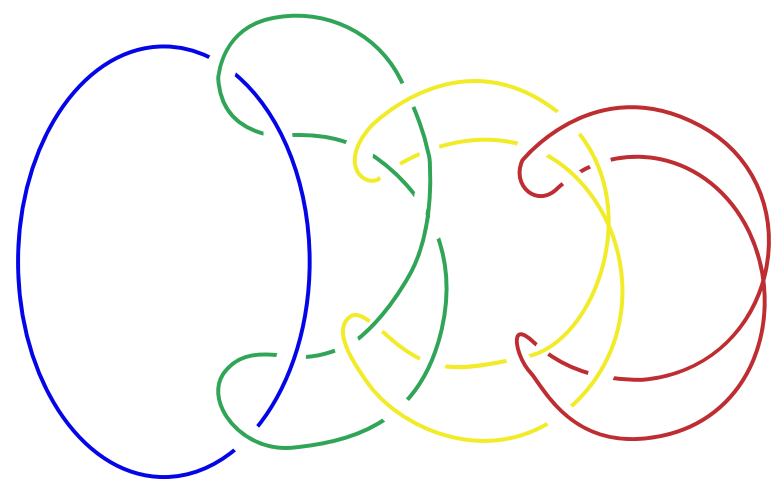

Dans la conférence à la Columbia University faite en décembre 75, Lacan (1975, p. 6) dit ceci : «Il faudrait s'émerveiller de la troisième dimension avant d'en faire une de plus. Il n'y a rien de plus facile que d'en faire une de plus. Quand les trois cercles s'en vont à la dérive, il suffit d'en faire un quatrième ».

\section{La particularité du nœud du Sinthome}

Ce nouage apparaît sous la plume de Lacan le 13 mai 75, dans la dernière partie de son séminaire RSI. 
Figure 3

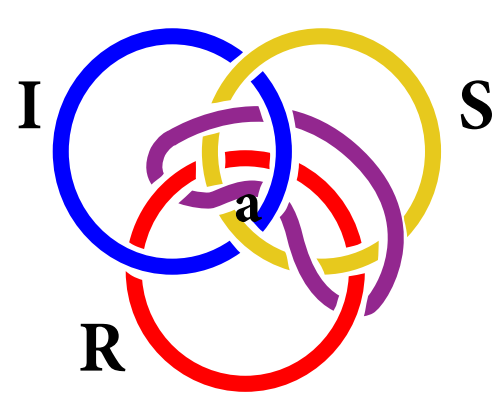

Ce que vous voyez dessiné là c'est un cercle qui d'une certaine façon redouble le cercle du réel. Lacan propose de considérer ça comme nomination du Réel, c'est-à-dire l'angoisse. L'angoisse fait le nœud en nommant le Réel.

Mais vous pouvez tout aussi bien redoubler le cercle de l'Imaginaire et vous aboutissez à la nomination de l'Imaginaire que Lacan considère comme inhibition. L'inhibition fait le nœud en nommant l'imaginaire.

Et enfin vous pouvez redoubler le rond du symbolique vous obtenez alors la nomination du symbolique que Lacan considère comme le symptôme. Le symptôme fait le nœud en nommant le symbolique.

Pourquoi Lacan considère-t-il que le quatrième rond équivaut à une nomination?

\section{La nomination implique le nom du père}

Il faut remarquer qu'il y a eu un changement de perspective concernant le nom du père dans l'enseignement de Lacan. Au départ, le nom du père, c'est le nom donné par la mère pour répondre à l'énigme de son désir, c'est le père nommé. Alors que dans les derniers séminaires, notamment dans RSI, le nom du père, c'est le nom donné par le père au fruit de son union avec la mère, c'est le père nommant et c'est aussi, par la même occasion, le père qui dit non. Lacan nous invite à considérer que le quatrième rond qui réunit les trois autres disjoints, assure cette fonction de nomination.

On peut se demander pourquoi ? En fait Lacan part du principe que dans la chaîne borroméenne à trois, les trois ronds s'équivalent complètement au point que si l'on n'utilise pas un artifice comme la couleur par exemple, on peut tout à fait les confondre. Ce n'est plus le cas dans la chaîne à quatre. Le quatrième rond s'amarre forcément à l'un des trois autres et de ce fait il le particularise. C'est en cela qu'on peut dire qu'il le désigne, donc qu'il le nomme.

Si le quatrième rond vient suppléer le rond du symbolique, s'il vient corriger une erreur d'écriture du nœud à ce niveau, Lacan parle du symptôme. 
Donc, concernant le symptôme, qu'il soit le nom du père ou autre chose, il assure donc la fonction de nomination.

A ce propos je vais vous parler du cas d'une patiente que j'ai rencontrée dans le cadre d'une présentation clinique. Il s'agit d'une femme qui ne se console pas du suicide de son compagnon survenu il y a neuf ans. Quelque chose a brisé son élan vital depuis cette date. Elle n'a pas fait le deuil de ce suicide dont elle se sent coupable. Malgré tout elle n'est pas purement mélancolique, car elle sait donner le change : «je porte un masque ». Elle se sent coupable, c'est sa façon de répondre à l'énigme du geste de son compagnon. «Pourquoi il a fait ça?»

Elle était en train de parler au téléphone avec sa belle-sœur, se plaignant de l'alcoolisme de son mari, elle aurait dit quelque chose comme : « il dit toujours qu'il va partir mais il ne le fait pas » là-dessus elle entend un bruit inhabituel ne s'en inquiète pas plus que ça mais, quelque temps plus tard, en cherchant son mari elle le retrouve pendu. Cet instant est fixé à tout jamais dans sa mémoire, elle revoit l'image en boucle. Curieusement, avec le peu d'explications qu'elle nous donne, on ne voit pas bien comment cet homme a pu faire pour se pendre, mais je me suis bien gardé de lui demander des détails. Le fait est qu'elle, elle voit la scène même si elle ne peut pas la décrire, elle ne cesse pas de la voir au présent.

Ce que nous avons su, c'est qu'elle avait choisi cet homme, $7^{\text {ème }}$ enfant d'une famille d'émigrés, parce qu'il avait l'air gentil et sans doute aussi parce qu'il représentait un trait d'exotisme. Mais nous avons appris qu'il avait été élevé par une mère seule, et on peut supposer que l'alcool lui servait à noyer mais à entretenir aussi sa douleur d'exister.

$\mathrm{Au}$ cours de l'entretien nous avons constaté que ce deuil impossible renvoyait à un autre deuil impossible du côté de ses parents et plus spécialement du côté de son père. Les parents avaient perdu leur fille ainée suite à une maladie grave et lorsque la patiente est venue au monde après le décès de l'ainée, le père a voulu donner à cette deuxième fille le prénom de l'ainée disparue. Dans sa propre famille, le père avait lui-même vécu cela, dit-elle, comme pour l'excuser. Dans son enfance elle se décrit sage comme une image. Mais à l'adolescence, tout change, elle se rebelle contre l'autorité paternelle et elle se met à vouloir écrire autrement son prénom, en en changeant une seule lettre, remplaçant un I par un Y. Avec ce petit changement de lettre seulement repérable par le passage à l'écrit, elle réussit à se démarquer de la sœur morte et donc à corriger le défaut de symbolisation de la mort que lui avait refilé son père.

Mais avec la mélancolie de son partenaire et son suicide, elle est rattrapée par cette question qu'elle avait subtilement éludée. "Depuis qu'il est mort, j'ai l'impression d'être devenue lui, je suis une morte vivante."

Elle qui s'était sans doute faite symptôme, qui croyait donc représenter l'objet, cause du désir de cet homme qui avait du mal à vivre, voilà que d'une minute à 
l'autre cette certitude s'effondre, le suicide du compagnon ruine cette idée et la renvoie à une question sans réponse : « qu'étais-je donc pour lui ? »

Dans la suite de cet entretien la question s'est posée du diagnostic quant à cet état dépressif, ce deuil qui se prolonge. S’agit-il d'une mélancolie ? Je ne le crois pas, quelque chose la préserve de cet effondrement, peut-être cette invention de la petite lettre qui l'a détachée de ce destin funeste transmis par le père.

Cette situation clinique un peu particulière a été commentée par Lacan (1960-1961, p. 215), dans le séminaire sur le transfert, il évoque ce genre de situation comme

(...) un point de concours entre deuil et mélancolie. Il s'agit d'un certain type de dénouement qu'il dit être de l'ordre du suicide de l'objet. Il s'agit de remords à propos d'un objet qui est entré à quelque titre dans le champ du désir et qui, de son fait, a disparu. Analysez ces cas, sondez ces remords dramatiques dans les cas où ils adviennent. Vous en trouverez la source dans ceci que cet objet, s'il est allé jusqu'à se détruire, ce n'était donc pas la peine d'avoir pris tant de précautions. Ce n'était donc pas la peine de m'être détourné pour lui de mon vrai désir.

On voit que la frontière est mince entre la valeur agalmatique de l'enfant mort - être celle qui manque, c'est s'assurer d'une valeur phallique - et la néantisation de l'objet qu'elle a été que lui renvoie le suicide de son compagnon. On peut penser que s'il lui a fallu se renommer elle-même en changeant une lettre au prénom donné par le père, c'est justement parce que d'avoir été nommée ainsi par son père ne l'assurait pas de sa valeur phallique mais la renvoyait déjà à cette position de l'objet réduit à néant.

On pourrait dire que son père lui a transmis une erreur au niveau du symbolique : la mort n'a pas été symbolisée correctement dans cette famille.

Son père l'a nommée comme sa fille ainée morte. Ça aurait pu donner à notre patiente une valeur phallique inaltérable. Mais dans l'hypothèse où il y aurait, au moins du côté paternel, une forclusion de la mort comme symbole, alors la nomination du père n'aura eu aucune autre valeur que celle de donner à sa deuxième fille le statut d'une morte vivante. C'est sans doute parce qu'elle a perçu que cette nomination ne valait rien qu'il lui a fallu s'auto nommer pour faire le nœud, ainsi elle a redoublé la nomination symbolique défaillante. On pourrait rajouter à ce registre du symptôme comme nomination le fait que cette patiente nous a confié que peu de temps après le suicide de son compagnon, elle a adopté une petite chienne à laquelle elle a donné un prénom féminin dont les deux premières lettres sont celles de son prénom. Cette petite chienne a une fonction importante car cet animal la maintient dans un lien social puisqu'elle fait commerce du toilettage et de l'éducation des chiens. 
Cette patiente qui a hérité d'une forclusion aurait pu déclencher une psychose. Elle ne l'a pas fait. Toute la discussion du cas a porté sur la question de savoir si ce deuil impossible était à considérer comme une mélancolie ou pas. Je ne le crois pas. Et je pense que la solution qu'elle a trouvée de suppléer à la nomination défaillante du père est à considérer comme son sinthome.

Je vais terminer sur le cas de Joyce dont Lacan s'est servi pour examiner les différentes façons de palier à une erreur du nœud.

Lacan élabore deux hypothèses concernant le cas Joyce.

Quand on regarde de près ce que Lacan a dit de Joyce, on s'aperçoit qu'il a élaboré deux hypothèses différentes. Mais je ne suis pas certain qu'il s'en soit aperçu lui-même.

La première hypothèse c'est de repérer dans la structure de Joyce une erreur du nœud qui conduit à ce que le réel et le symbolique se nouent en laissant l'imaginaire à la dérive. Lacan situe l'épisode de la raclée décrite dans le portrait de l'artiste en jeune homme comme un témoignage du rapport de Joyce à son corps pas affecté par les coups mais glissant sur lui comme une pelure.

Quant au nœud entre le réel et le symbolique, Lacan en repère la conséquence dans les fameuses épiphanies de Joyce. Vous savez que ce sont des signifiants articulés de façon bizarre qui rappellent à Joyce des moments de perplexité qu'il a vécus et qui ressemble beaucoup à ce que nous appelons des " phénomènes élémentaires » et qui sont pour la nosographie psychiatrique classique des prodromes du déclenchement de la psychose. Ces épiphanies lient la rencontre avec le réel innommable à certains signifiants. Ces épiphanies n'ont aucun sens pour le lecteur. Joyce s'est efforcé tout au long de sa carrière d'écrivain d'essayer d'insérer ces bouts de phrases dans son texte pour les amarrer à un sens. On peut donc considérer qu'il s'agit d'un nouage particulier du réel avec le symbolique.

Dans cette hypothèse on peut remarquer que Joyce a hérité d'un nouage plutôt olympique, ce nœud est voisin de celui que Lacan attribue au petit Hans chez lequel, le réel est noué avec l'imaginaire, laissant le symbolique à la dérive. La phobie viendrait l'amarrer aux deux autres.

Avant d'examiner la seconde hypothèse concernant le nœud chez Joyce, il faut que je vous présente le nœud de trèfle. C'est le premier des nœuds premiers. Il existe des nœuds premiers comme il existe des nombres premiers.

Pour transformer la chaîne borroméenne en nœud de trèfle il faut lui faire subir un petit traitement qui consiste à sectionner les ronds en $\mathrm{D}, \mathrm{E}$ et $\mathrm{F}$,

La chaîne borroméenne à trois répond au même principe mathématique que le nœud de trèfle. 
Figure 4

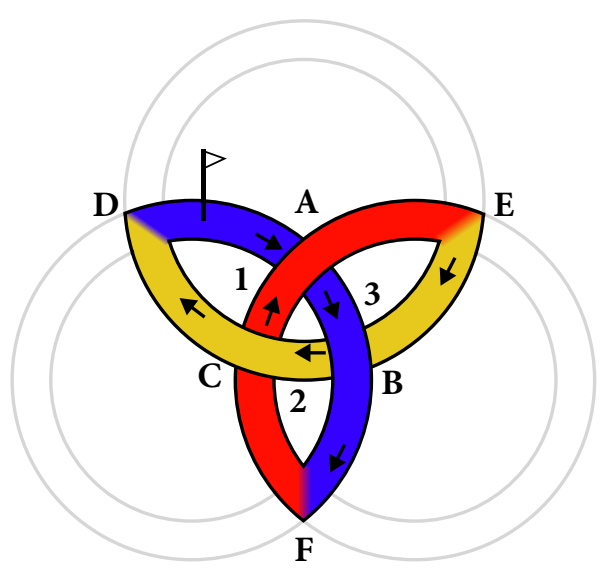

Si vous faites cette opération sur trois ronds qui ne sont pas enchainés de façon borroméenne, vous obtiendrez un faux nœud de trèfle, autrement dit un nœud trivial qui se réduit à un rond.

Dans la seconde hypothèse du nœud de Joyce qu'on peut déduire d'un schéma que Lacan donne du faux nœud de trèfle on peut supposer que si le centre de la chaine borroméenne qui caractérise Joyce est un faux nœud de trèfle, alors, c'est que chez lui les trois ronds sont disjoints et ce n'est pas tout à fait la même chose.

Lacan envisage alors plusieurs façons de réparer l'erreur du faux nœud de trèfle soit en corrigeant l'erreur là où elle s'est produite ou bien ailleurs. On n'obtient pas le même résultat. 



\section{Le nœud borroméen et l'écriture de la clinique psychanalytique (17/02/2019)}

Qu'est-ce qui s'écrit de l'expérience analytique? Pas grand-chose d'intéressant, rien qui puisse faire littérature, mais pourtant quelque chose devrait pouvoir s'en écrire et c'est ce que nous nous efforçons de faire quand nous construisons un cas, quand nous réfléchissons sur une présentation clinique. Bien souvent il se produit quelque chose à un moment du débat entre nous, quelque chose qui, après s'être construit peu à peu, prend tout à coup une allure d'évidence. C'est ça! Eh bien ce «c'est ça! » témoigne que quelque chose vient de s'écrire de la logique que nous avons repérée, la logique du trajet particulier d'un sujet.

Il faut dire que les cas que l'on nous soumet dans les constructions ou lors des présentations cliniques sont des cas difficiles. Nos catégories classiques de névrose psychose ou perversion ne suffisent pas à dégager la logique du cas. Par contre, je remarque que j'utilise de plus en plus souvent la logique borroméenne pour m'y repérer et je vous propose donc de réfléchir avec moi sur cette expérience d'une possible écriture borroméenne de la clinique.

Lacan (1977, p. 7) disait que « la clinique c'est le réel en tant qu’il est impossible à supporter ». C’est pour ça qu'il est légitime de vouloir écrire, esquisser ce réel. Et l'outil borroméen parait adéquat à cette tâche qui reste à la limite du possible puisqu'il s'agit du réel.

Plusieurs fois dans son séminaire sur Les non-dupes... Lacan évoque une écriture qui ne soit pas à lire, une écriture du réel qui serait l'écriture du nœud borroméen.

Le nœud borroméen écrit l'articulation entre les trois dit-mensions de l'espace habité par l'être parlant. Parler de dit-mension implique le dire et donc le temps et l'évènement.

Ce que le nœud opère et ce qu'il écrit, c'est un coincement. La logique du nouage fait que si deux dimensions glissent l'une sur l'autre sans s'arrêter, il faut une troisième placée de la bonne façon pour coincer ce glissement infini ou éternel. Le nœud met un coup d'arrêt à cette éternité. Quelque chose cesse de ne pas s'écrire et fait évènement.

Le nœud, comme écriture de l'évènement implique le temps. Lacan nous suggère que le nœud montre les «tiraillements» du temps. Tiraillement entre le symbolique où s'inscrit le passé, entre le futur que l'on imagine et le réel du présent qui nous file entre les doigts. Ce nœud subtil permet à chacun de se débrouiller du présent, de l'écrire en histoire et donc de l'oublier. Certains phénomènes cliniques comme la mémoire traumatique pourraient s'envisager sous l'angle d'un défaut de ce noud du temps. 


\section{La mémoire traumatique}

Il y a deux ans à Sao Paulo, j'ai donné une conférence sur l'écriture du temps avec la chaine borroméenne en commentant à ma façon une phrase extraite du Livre XI des Confessions de Saint Augustin. Toute son argumentation repose sur le fait que le passé et l'avenir n'existent que comme représentations dans le discours du présent. Ça l'amène à cette formulation: «il y a trois temps : le présent du passé, le présent du présent, le présent du futur. Le présent du passé, c'est la mémoire ; le présent du présent, c'est l'intuition directe; le présent du futur, c'est l'attente ».

Cette subtile structuration trinitaire du temps chez Augustin combinée aux trois ek-stases de la temporalité chez Heidegger m'a conduit à inscrire cette structuration de la temporalité selon les trois registres avec lesquels nous nous orientons dans la réalité : le Symbolique, L'Imaginaire et le Réel. Je proposais d'assimiler le passé à la catégorie du Symbolique, puisqu'il est fait de représentations qui s'organisent en histoire. Le futur, en tant qu'il n'a d'existence qu'à être imaginé est bien sûr à assimiler à l'imaginaire. Il nous reste à admettre que le présent est assimilable au réel. C'est admissible, c'est pourquoi il fuit en permanence, il n'est qu'en cessant d'être comme dirait Augustin.

Donc si le présent est assimilable au réel, on comprend que le névrosé cherche à le fuir. Pourtant, être ouvert au présent, a quelques vertus, c'est savoir profiter de la contingence, c'est savoir profiter de la réalité. Etre dans la réalité, c'est ce que l'on peut souhaiter de mieux à l'être parlant. C'est pouvoir aborder le présent du réel, avec le présent du passé symbolique, c'est-à-dire nos représentations, nos souvenirs, et le présent du futur imaginaire, c'est-à-dire nos attentes, nos désirs. Catégoriser ainsi le présent réel, le passé symbolique et le futur imaginaire me conduit à inscrire cette construction trinitaire du temps subjectif à l'aide de trois cercles d'Euler représentant les trois catégories du présent du passé et du futur.

Figure 5

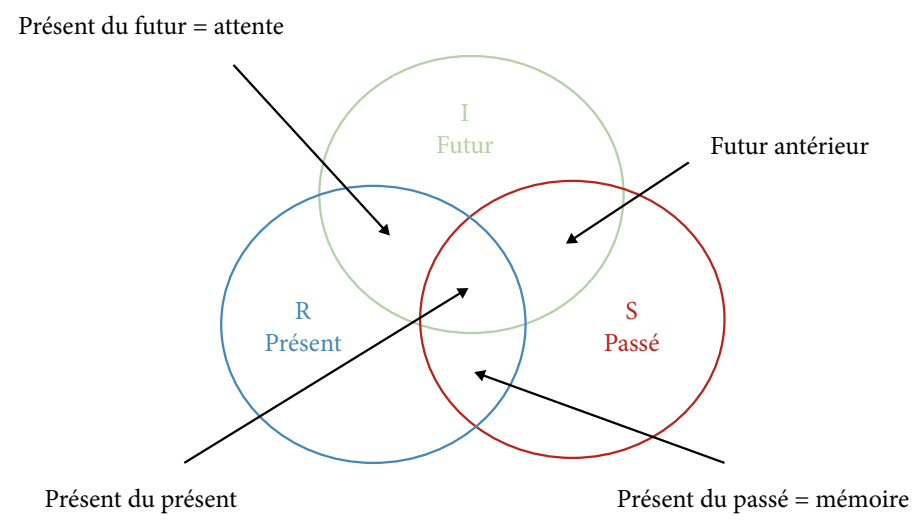


A l'intersection du présent et du passé, vous pouvez inscrire le présent du passé, c'est-à-dire la mémoire, les souvenirs.

A l'intersection du présent et du futur, Augustin nous conseille d'inscrire l'attente et là on peut y mettre aussi bien le désir, quand on attend des bonnes choses, que l'angoisse quand on imagine le pire.

Reste à voir ce que l'on pourrait inscrire dans l'intersection entre le passé et le futur. A priori, on ne voit pas, en dehors du fait que le passé et le futur s'articulent en partie grâce au présent du présent.

Mais il y a bien une articulation qui existe entre le passé et le futur, en dehors du présent, c'est l'hypothèse du futur antérieur, ce qui vous fait dire «il aura été ». C'est un coincement particulièrement important du temps car c'est lui qui détermine la signification, après-coup, d'un évènement. La notion d'après-coup soulignée par Freud montre que le discours peut produire des effets de significations qui peuvent modifier des représentations du passé, réorganiser des souvenirs, et même créer des traumatismes après-coup.

J'entendais récemment Boris Cyrulnik, interrogé sur la mémoire traumatique, dire cette chose simple : le traumatisé souffre deux fois, il souffre du coup et de la représentation du coup. En définitive, la mémoire traumatique témoigne d'un passé qui ne passe pas, c'est-à-dire qui ne s'oublie pas, qui reste dans ce statut particulièrement réel du présent du présent augustinien.

Dire que le passé passe, c'est dire qu'au moment présent il n'est plus là, il est naturellement oublié même s'il est inscrit quelque part, et c'est justement parce qu'il s'est inscrit quelque part en terme de traces mnésiques qui ont pris du sens, qui se sont inscrites dans une histoire que le passé passe et reste à sa place.

En somme cette écriture du nœud du temps que je vous propose, c'est une écriture qui noue le réel du présent, au symbolique du passé et à l'imaginaire du futur. Il faut pouvoir faire ce nœud pour se construire une histoire et pouvoir s'accommoder de la réalité présente.

Vous aurez remarqué que quand tout va bien, quand on a un projet, quand on sait où l'on va, quand le passé reste à sa place et ne nous oblige pas à ressasser, eh bien on ne voit pas le temps passer. C'est-à-dire que l'on oublie qu'il passe. Alors que, si vous interrogez un mélancolique, il vous dira que pour lui le temps s'éternise, il ne passe pas, il ne peut pas l'oublier ; le mélancolique ne peut pas oublier son être pour la mort, c'est à dire qu'il ne pense qu'à mourir. Cioran disait fort justement que pour lui, au plus fort de ses moments de mélancolie, «le temps ne s'abaisse pas à l'évènement. " En s'inscrivant dans l'évènement, le temps comme objet réel s'oublie, il passe sans qu'on s'en aperçoive, sans qu'on s'en inquiète trop.

Pour revenir à la question de la mémoire traumatique, on pourrait dire qu'elle témoigne de quelque chose qui ne passe pas à l'écriture de l'évènement. C'est-à-dire que c'est la répétition de quelque chose qui reste réel, atrocement présent. 
C'est une page qui ne se tourne pas et si l'on y réfléchit bien, c'est une page sur laquelle rien n'a pu s'écrire.

Freud ne disait pas autre chose quand il essayait de comprendre la signification des rêves traumatiques. Si ce genre de rêves se produisent et se répètent, c'est pour reproduire le trauma et essayer de le dominer, c'est-à-dire de le faire passer à une écriture symbolique. On pourrait concevoir la mémoire-répétition, autrement dit, la mémoire traumatique comme un nouage particulier du réel du présent au symbolique du passé, ou pour être plus précis, c'est comme si le réel du présent et le symbolique du passé étaient en continuité, comme si l'on passait de l'un à l'autre sans coupure ni nœud.

Cette hypothèse cadrerait bien avec ce que l'on appelait à l'époque du Quattrocento : arte dell'oblio. On décrivait ainsi un remède contre l'envahissement de cette mémoire répétitive. On prescrivait au patient de traduire en image le souvenir importun, de tracer l'image sur un papier que l'on froissait que l'on déchirait, que l'on brulait, ou que l'on jetait dans l'eau courante. Il s'agissait donc de réintroduire l'imaginaire pour permettre un autre lien entre le réel et le symbolique, un passage par l'écriture pour éteindre ce phénomène répétitif.

Je pratique depuis longtemps, sans le savoir, cet art de l'oubli dans la cure avec des enfants. Quand ils me parlent de cauchemars répétitifs, je les invite à essayer de les dessiner ; ça fait généralement surgir des éléments signifiants dont j'essaye de leur faire entendre la valeur équivoque, ce qui souvent les fait rire et déplace l'accent terrorisant. La plupart du temps, c'est très efficace, la répétition du cauchemar cesse. Je comprends aujourd'hui pourquoi : c'est que je propose le passage par l'image pour nouer autrement le réel au symbolique. Quelque chose s'écrit et peut alors passer à l'oubli. Ça cesse parce qu'enfin ça s'écrit. Lacan définissait ainsi la catégorie modale du possible : ce qui cesse, de s'écrire. C'est ce qui se distingue du nécessaire qui ne cesse pas de s'écrire et c'est ce qui s'oppose radicalement à l'impossible qui ne cesse pas de ne pas s'écrire.

Dans la littérature psychiatrique on peut lire le cas d'un patient du neuropsychiatre russe le Dr. Alexandre Romanovitch Luria, un patient atteint d'hypertrophie de la mémoire qui en tirait un bénéfice substantiel en se présentant en public comme mnémoniste professionnel. Lui qui se produisait plusieurs fois par soirée avait besoin de se ménager des plages d'oubli. Il avait mis au point une stratégie : il notait par écrit ce qu'il voulait oublier. Lorsque cette astuce ne suffisait pas à effacer le souvenir importun, il déchirait le papier le brûlait ou le jetait à l'eau. Vous remarquerez que ce patient utilise le même stratagème que celui que proposait l'arte dell'oblio. Mais en même temps on voit que stratagème utilise l'écriture qui normalement sert à ne pas oublier. Tous ceux qui cherchent à renforcer leur mémoire utilise aussi des stratagèmes de cette sorte. Les sujets atteints $\mathrm{du}$ syndrome d'Asperger qui sont condamnés à ne rien oublier en témoignent facilement : Il s'agit toujours d'associer un chiffre, à une couleur, à une image. Ce qui est frappant, c'est de constater que l'effort fait pour mémoriser un signifiant qui 
consiste à l'accrocher à une image, est du même ordre que l'astuce préconisée par l'arte dell'oblio pour se débarrasser d'un signifiant importun. Ce qui nous amène à considérer que mémoire et oubli sont sans doute les deux faces d'un même processus qui met en jeu l'écriture, c'est-à-dire une façon d'accrocher les signifiants dans un réseau selon les trois coordonnées essentielles de la structure : le Réel, le Symbolique et l'Imaginaire, ce qui réalise l'écriture d'une histoire et fait le tri entre ce qui se passe, ce qui s'est passé et ce qui va se passer.

L'inconscient freudien fait précisément le joint entre mémoire et oubli. Ce qui est refoulé est bien écrit quelque part. Et c'est même parce qu'il y a un nœud d'écriture, parce qu'un signifiant est pris dans une chaîne, qu'il est retenu captif et qu'il n'est pas mobilisable pour la remémoration.

Le refoulement ne consiste pas en un effacement de la trace. Freud (1901/1991) a consacré une bonne partie de sa Psychopathie de la vie quotidienne à l'oubli. Dans le cas célèbre de l'oubli du nom Signorelli, par exemple, il montre comment ce nom reste prisonnier d'une chaîne de signifiants qui l'associe à des pensées que Freud voudrait oublier, notamment des questions concernant la sexualité et la mort. C'est par une elation métonymique que le nom de Signorelli est retenu hors de portée de la remémoration. Il est retenu dans un nœud de représentations interdites.

C'est à dessein que j'utilise ce signifiant retenu, car il prête à équivoque ; à la fois il dit que ce signifiant n'est pas disponible et en même temps, dire qu'il est retenu signale bien qu'il n'est pas effacé pour autant, qu'il est pris dans une mémoire qui n'est rien d'autre que l'inconscient. La plupart des exemples que Freud nous livre dans sa Psychopathologie de la vie quotidienne, à propos de ces oublis de nom, montrent qu'un nom est oublié parce qu'il est retenu dans un nœud d'associations. Quelque fois il nous montre qu'à la place du nom oublié une image s'impose. C'est le cas d'ailleurs de l'oubli du nom Signorelli. Freud remarque que moins il peut se souvenir du nom et plus la figure du peintre s'impose dans son souvenir. Il voit l'autoportrait du peintre qui s'est représenté lui-même dans l'une de ses fameuses fresques. Quand son compagnon de voyage lui souffle le nom du peintre qui lui manquait Freud remarque que l'image de l'autoportrait s'estompe. On pourrait en déduire que l'image servait à cacher le nom à oublier. Freud remarque d'ailleurs que ses souvenirs d'enfance ont toujours un caractère visuel. Ceci l'amènera à étudier la question du souvenir-écran, question particulièrement intéressante puisqu'elle combine un signifiant oublié et une image. Remarquons simplement la fonction de l'image dans ce nœud fait par l'inconscient pour retenir dans l'oubli une représentation censurée par la conscience.

Pour oublier, l'inconscient retient certains signifiants dans son écriture. L'inconscient écrit pour que le sujet puisse oublier.

Mais il y a de l'inoubliable, c'est toute la question du traumatisme. Comment faire passer ça à l'inconscient? 
A ce propos je pourrais évoquer un souvenir, pour moi inoubliable, celui d'une petite fille de 5 ou 6 ans qu'on m'avait présentée à Medellin en Colombie. Sa psychologue voulait avoir mon avis sur la conduite à tenir face à cette petite fille qui avait été exclue de l'école pour des troubles du comportement. Il faut dire que son comportement était particulièrement inadapté puisqu'elle proposait aux petits garçons de leur faire des fellations. Elle était tellement ingérable que l'école n'avait pas trouvé d'autres solutions que de l'exclure. Il faut dire que cette petite fille était élevée par une mère célibataire qui se livrait à la prostitution à son domicile.

Je ne me souviens plus exactement de ce que cette fillette a pu me dire. Elle avait surtout des questions à me poser sur des choses simples de la vie quotidienne qui trahissaient le fait de son manque de repères. Ce dont je me souviens parfaitement, par contre, c'est de la phrase sur laquelle s'est terminé notre entretien : « je voudrais que tu m'apprennes à écrire ».

Nous aussi nous devons apprendre à écrire à partir du réel de la clinique et je vais essayer maintenant de vous montrer l'outil dont je me sers pour apprendre à écrire.

Pour pouvoir se servir correctement de l'écriture borroméenne il faut en dégager les principes.

Le principe essentiel est que chaque dit-mension - que Lacan appelle aussi consistance - peut servir de moyen pour nouer les deux autres. Aucune consistance n'a de privilège à cet égard. Concernant la chaine borroméenne à trois, c'est parce que deux ne sont pas nouées qu'une troisième, quelqu'elle soit, peut faire le nœud. Concernant la chaîne à quatre, c'est parce que trois ne sont pas nouées qu'une quatrième peut faire le nœud.

Le problème, c'est qu'étant donné qu'il n'y a que trois consistances, le quatrième rond sera donc forcément un doublement de l'une des trois autres. Lacan résout cette difficulté en désignant le quatrième soit comme nomination - symbolique, imaginaire ou réelle - soit comme symptôme.

Dans son livre sur les Lettres du symptôme Erik Porge dégage un théorème efficace en disant que chaque chaîne à $n$ consistances peut être considérée comme réparation d'un nœud fautif à $n-1$ consistances. Ainsi, la chaîne à trois pallie au fait que deux ne sont pas nouées, la chaîne à quatre pallie au fait que trois ne soient pas nouées et ainsi de suite. Ce point m’apparaît important car il permet de voir que le borroméen commence à trois et que le nœud à quatre n'en est qu'une version, il obéit à la même logique.

Un second principe est que si les trois consistances sont équivalentes au niveau de leur fonction de nouage, le fait de se servir des trois anneaux de l'armoirie des Borromée pour désigner, en les distinguant, les trois consistances symbolique imaginaire et réelle, nous oblige à nous y repérer le plus justement possible dans l'expérience clinique sur ce qui est de l'ordre symbolique, sur ce qui est de l'imaginaire et sur ce qui reste de l'ordre du réel. 
Si dans un repérage clinique traditionnel nous nous efforcions de repérer, au niveau des rapports de chaque sujet avec la chaîne signifiante qui le constitue, la métaphore paternelle ou sa forclusion, la fonction phallique, les points de capitons, la métonymie. Ici, pour pouvoir écrire en termes borroméens les éléments de l'histoire de chacun et les moments cruciaux de son trajet dans une cure analytique, nous devons savoir les catégoriser, sans préjugé aucun, dans les trois ordres symbolique, imaginaire ou réel.

Un troisième et dernier principe que je vous propose, c'est qu'il faut connaître le maniement de la chaîne borroméenne. Connaître ses principaux ratages, en mesurer les conséquences et connaître les réparations possibles. On entre là, un peu, dans ce que j'appelle : le solfège du nœud.

Figure 6

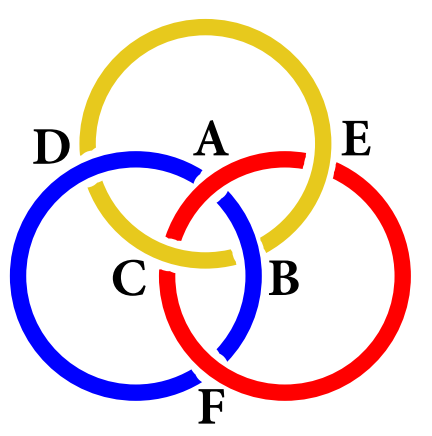

La chaîne borroméenne à trois comporte 6 points de croisement. Mais pour chaque rond il y a 4 points de croisement avec les deux autres. Chacun de ces croisements répond à une alternance de dessus dessous et pour respecter la logique borroméenne chacun des ronds doit croiser les deux autres de la même façon — soit toujours dessus, soit toujours dessous - sinon deux ronds vont se nouer entre eux, ce qui est exclu.

Puisque nous voulons favoriser un travail d'atelier, je vais vous soumettre un petit exercice auquel je me suis livré pour m'y repérer dans les principaux ratages du nœud et mesurer leur conséquence.

Remarquez qu'il y a des points de croisement centraux - je vais les appeler A, $\mathrm{B}, \mathrm{C}$ - puis des croisements périphériques - appelons les $\mathrm{D}, \mathrm{E}, \mathrm{F}$.

Commençons par étudier les erreurs en périphérie.

Erreur au point $F$ : Le rond rouge après être passé sous le jaune, passe sous le bleu au lieu de passer dessus en respectant la succession dessous dessus. Si vous regardez bien, cette erreur libère le rond jaune. Autre conséquence, le rouge et le bleu s'interpénètrent. 


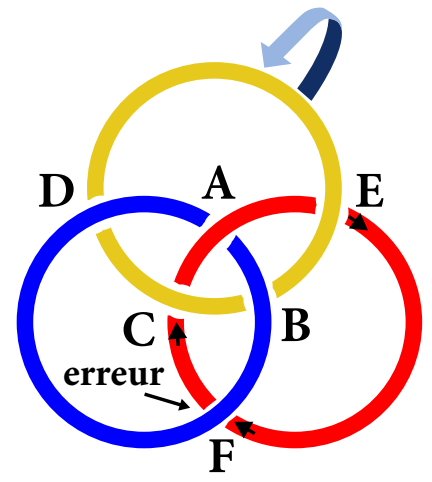

Pour les points $E$ et $D$ il en va de même : une erreur au point $E$ libère le rond bleu et enchaîne le rouge et le jaune. Une erreur au point $\mathrm{D}$ libère le rond rouge et enchaîne le bleu au jaune.

Maintenant, examinons les erreurs au centre. C'est plus facile à voir.

Erreur au point $A$ : Le rond rouge après être passé sous le jaune, passe encore sous le bleu. Cette erreur libère le rond jaune et rouge et bleu s'interpénètrent. C'est l'erreur que Lacan attribue au cas Joyce, c'en en est au moins une version car si vous lisez attentivement le séminaire sur le sinthome vous constaterez que Lacan donne, sans forcément s'en apercevoir, deux versions distinctes du nœud fautif chez Joyce.

Je ne détaille pas les erreurs en $\mathrm{B}$ ou $\mathrm{C}$, elles répondent à la même logique : libération d'un rond, enchainement des deux autres.

Figure 8

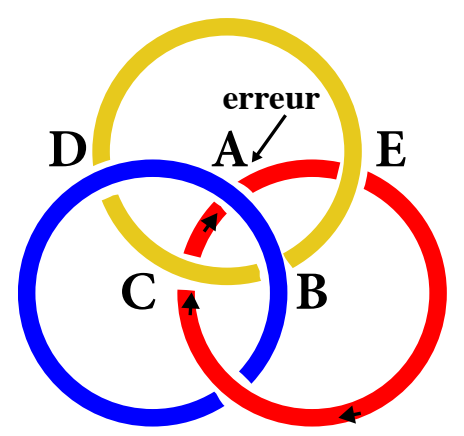

En suivant, je me suis mis en devoir d'étudier les conséquences de la conjugaison de deux erreurs au centre et à la périphérie. 
Erreur en $A$ et $F$ : Vous voyez tout de suite que les trois ronds se libèrent.

Figure 9

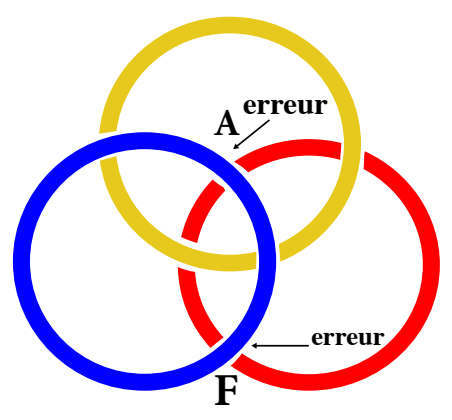

Erreur en $E$ et $C$ ou en $B$ et $D$ : C'est la même chose, les trois ronds se libèrent. Seule différence, c'est l'ordre de superposition des trois ronds, mais elle est strictement sans conséquence.

On peut en conclure qu'un rond reste coincé à condition que les deux croisements dans lesquels il n'est pas directement impliqué ne soient pas fautifs : par exemple croisement $A \& F$ pour le rond jaune, croisement $B \& D$ pour le rond rouge et croisement $\mathrm{C} \& \mathrm{E}$ pour le rond bleu. C'est un point intéressant car ces points névralgiques sont ceux qui définissent les trois espaces que Lacan nomme : jouissance phallique - concernant $\mathrm{A} \& \mathrm{~F}$, sens - concernant C\&E et jouissance de l'Autre - concernant B\&D. C'est le coinçage résultant de l'entrelacement de ces trois jouissances qui assure le nouage borroméen.

Il y a d'autres combinaisons d'erreurs possibles, elles aboutissent à des nœuds complexes qui ne sont pas borroméens ni pas vraiment olympiques non plus. Il existe un tableau des nœuds premiers auquel il faudrait se référer pour pouvoir définir ces nœuds-là, mais c'est un très gros travail auquel je n'ai pas eu le temps de me livrer.

Ce que je retiens, c'est que l'écriture d'un nœud borroméen ne se rate pas si facilement que ça. J'ai dû faire des efforts pour inscrire ces ratages. C'est intéressant comme expérience parce que ça semble contredire ce que Lacan nous en dit, car lui, dans ses premiers pas de la logique du nœud, passait beaucoup de temps à se tromper dans leur écriture. Je ne sais pas ce que je peux tirer de ce constat mais c'est comme ça.

Quoiqu'il en soit, un nœud borroméen, ça peut se rater. Et ce n'est pas forcément un drame, ça peut donner l'occasion au sujet à qui l'on a transmis l'écriture de ce ratage d'en trouver une solution. 
Si j'évoque une transmission possible du ratage du nœud, c'est parce que Lacan (1973/1974, p. 69) le suggère dans une petite remarque à propos de la succession. Il glisse de l'écriture de la succession des dessus dessous dans la chaîne borroméenne, pour obtenir la bonne forme, à ce que traitent les notaires dans les affaires de succession, c'est-à-dire l'héritage. Et c'est là qu'il parle du «titre de noblesse, l'ancienneté de la famille, qui est, pour le généalogiste, toujours trouvable, pour n'importe quel imbécile et donc aussi pour n'importe quelle imbécillité ».

Il est certain qu'on hérite d'un titre de noblesse qui vaut comme reconnaissance purement symbolique mais il n'y a pas besoin de fouiller très loin dans l'Histoire pour démontrer que la transmission d'un titre de noblesse ne garantit pas que cet héritage ne se soit pas accompagné de la transmission d'un ratage du nœud. Les exemples où le titre de noblesse couvre le ratage du nœud ne sont pas rares.

Depuis que Lacan a découvert la chaîne borroméenne la mathématique des nœuds a évolué. On sait maintenant écrire un nœud avec une formule mathématique.

Pour aborder ce système d'écriture on peut partir du premier des nœuds, c'est-à-dire le nœud de trèfle.

C'est à propos de ce nœud que l'on peut commencer à envisager un solfège du nœud, c'est-à-dire une écriture mathématique de la succession des croisements rencontrés quand l'on s'imagine parcourir le nœud. On choisit un point de départ et on voit que l'on rencontre d'abord un tunnel 1 , puis un pont, un deuxième tunnel 2, puis un pont et enfin un troisième tunnel 3, un dernier pont et on est arrivé. On a rencontré donc 6 croisements : 3 tunnels et 3 ponts. On caractérise chaque tunnel par le pont qui le surplombe et le sens du trajet sur ce pont selon que sur ce pont on passe de gauche à droite ou de droite à gauche. On obtient ainsi la matrice du nœud qui servira de base à l'écriture d'un polynôme, c'est-à-dire une écriture logique qui rend compte de la nature du nœud. Et puisque le nœud est réel, on a donc une écriture du réel. On comprend pourquoi Lacan s'est acharné à dégager la logique du nœud. C'est ce point à l'horizon qu'il visait en cherchant à élaborer une écriture qui rende compte de la clinique psychanalytique.

Ce qui est important pour nous dans ce nœud de trèfle qui est au cœur de la chaîne borroméenne, c'est qu'il correspond à l'entrelacement des trois sortes de jouissances que Lacan situe au cœur de la chaine borroméenne. Chaque principe de jouissance tirant de son côté, le nœud se coince autour de l'objet $a$ qui se trouve au centre et ce coincement évite qu'un principe de jouissance l'emporte sur les deux autres. 


\section{Références bibliographiques}

Freud, S. (1991). Psicopatología de la vida cotidiana (sobre el olvido, los deslices en la habla, el trastrocar las cosas confundido, la supertición y el error). In S. Freud. Obras completas de Sigmund Freud (Vol. VI, pp. 1-284). Buenos Aires : Amorrortu. (Texte original publié en 1901)

Lacan, J. (1960-1961). Séminaire 8: Le Transfert. Retrouvé en 15 août, 2019, de http:// staferla.free.fr/S8/S8\%20LE\%20TRANSFERT.pdf

Lacan, J. (1971-1972). Séminaire 19 : ...Ou pire. Retrouvé en 15 août, 2019, de http:// staferla.free.fr/S19/S19...OU\%20PIRE.pdf

Lacan, J. (1973-1974). Les Non-dupes errent. Retrouvé en 15 août, 2019, de http:// staferla.free.fr/S21/S21\%20NON-DUPES....pdf

Lacan, J. (1974-1975). R.S.I. Retrouvé en 15 août, 2019, de http://staferla.free.fr/S22/ S22\%20R.S.I..pdf

Lacan, J. (1975). Conférences et entretiens dans des universités nord-américaines. Retrouvé en 15 août, 2019, de http://ecole-lacanienne.net/wp-content/ uploads/2016/04/1975-12-01.pdf

Lacan, J. (1975-1976). Le Ainthome. Retrouvé en 15 août, 2019, de http://staferla.free. fr/S23/S23\%20LE\%20SINTHOME.pdf

Lacan, J. (1977). Ouverture de la section clinique. Retrouvé en 15 août, 2019, de http:// traco-freudiano.org/tra-lacan/abertura-secao-clinica/abertura-clinica.pdf

Porge, E. (2010). Lettres du symptôme : versions de l'identification. Toulouse: Erès.

Santo Agostinho (2001). Confissões. Lisboa : IN-CN. Retrouvé en 15 août, 2019, de http://www2.uefs.br/filosofia-bv/pdfs/agostinho_01.pdf

Recebido: $15 / 01 / 2020$

Aprovado: 15/01/2020 
